\title{
On the Polar Nature and Invariance Properties of a Thermomechanical Theory for Continuum-on-Continuum Homogenization
}

\author{
Kranthi K. MANDADAPU ${ }^{1,2 *}$, B. Emek ABALI ${ }^{3}$, and Panayiotis PAPADOPOULOS ${ }^{3 \dagger}$ \\ ${ }^{1}$ Department of Chemical and Biomolecular Engineering, University of California, Berkeley, CA 94720-1462, USA \\ ${ }^{2}$ Chemical Sciences Division, Lawrence Berkeley National Laboratory, Berkeley, CA 94720-1740, USA \\ ${ }^{3}$ Department of Mechanical Engineering, University of California, Berkeley, CA 94720-1740, USA
}

Table of contents

1 Introduction $\quad 2$

2 Overview of the Extensive Homogenization Method 4

2.1 Review of previous results: balance of mass and linear momentum . . . . . . . . . 4

2.2 Homogenization of angular momentum . . . . . . . . . . . . . . . . . 5

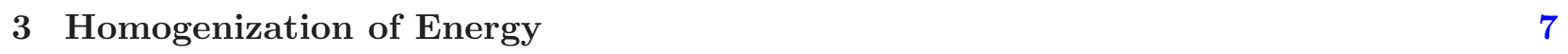

4 Invariance of Extensive Relations $\quad 10$

5 Invariance: Macroscopic Cauchy Stress and Linear Momentum Balance 15

6 Invariance: Couples and Angular Momentum Balance 16

7 Invariance: Macroscopic Heat Flux and Energy Balance 17

8 Conclusions $\quad 17$

Appendix A Derivation of the Macroscopic Energy Balance Equation 22

Appendix B Divergence-free Terms in the Macroscopic Cauchy Stress 23

\begin{abstract}
This paper makes a rigorous case for considering the homogenized continuum derived by the Irving-Kirkwood procedure as a polar medium in which the balances of angular momentum and energy contain contributions due to body couples and couple stresses defined in terms of the underlying microscopic state. The paper also addresses the question of invariance of macroscopic stress and heat flux and form-invariance of the macroscopic balance laws.
\end{abstract}

Keywords: continuum homogenization; extensivity; Irving-Kirkwood procedure; polar media; invariance

\footnotetext{
*kranthi@berkeley.edu (corresponding author)

†panos@berkeley.edu (corresponding author)
} 


\section{Introduction}

Continuum-on-continuum homogenization provides a convenient theoretical framework for analyzing media in which there exists sufficient length- and time-separation between the macroscopic body and its microstructural components, while, at the same time, both may be accurately modeled as continuous media. This may well be the case for bulk metals (with their polycrystalline microstructure) and composites (with, say, their matrix-fiber microstructure). In the general thermomechanical setting, the goal of homogenization theories is to deduce (homogenized) macroscopic counterparts for all the kinematic and kinetic variables that enter the microscopic description of the continuous medium.

The pioneering work of Irving and Kirkwood [1] on the upscaling of classical statistical mechanics to continuum hydrodynamics motivated a recent study of continuum-on-continuum homogenization, which led to the rigorous derivation of formulae for macroscopic stress and heat flux based on a minimal set of assumptions, that is, extensivity of mass, momentum, and energy [2]. While phase-space averaging was substituted by mass-weighted volume averaging and interacting particles in the microscale were replaced by a continuum, the critical dependence on extensivity and the procedural similarity in the derivations render the continuum-on-continuum homogenization method in [2] a close relative to the original Irving-Kirkwood method. The resulting formulae incorporate naturally the volumetric effect of inertia on both stress and heat flux and can be used in practical computations using, e.g., two-scale finite element methods [3]. While it can be plausibly assumed that at appropriately small length scales such volume effects become negligible compared to surface effects, as is argued in the continuum homogenization literature (see, e.g., [4]), volumetric effects become dominant in the presence of non-trivial velocity fluctuations, as is the case with wave propagation in heterogeneous media where wavelengths are in the order of the length scale $[5,6,7]$. It is important to note that continuum homogenization theories based on extensivity have been already considered in other field theories, such as electrodynamics [8].

The present paper explores the polar nature of the macroscopic continuum in the homogenization theory, motivated intuitively by the premise that the length scale of the underlying microstructure is generally bound to yield non-vanishing body and surface couples. The polar nature is established methodologically by the approach adopted in [9, 10] for upscaling atomistic systems with internal couples to the continuum hydrodynamics. In particular, it is shown that the distinction between macroscopic angular momentum and moment of momentum, argued masterfully in [11], albeit with only a general allusion to directed media, is a natural implication of the homogenization theory. In 
fact, it is rigorously confirmed that couple forces, defined in terms of the microscopic state, enter in a non-trivial statement of macroscopic angular momentum balance. The proposed theory differs from the micromorphic theory $[12,13]$ both methodologically and philosophically. Indeed, the micromorphic theory relies on homogenization rules for kinetic quantities, such as stress and heat flux, which are not extensive. In addition, constitutive laws for the micromorphic continuum are postulated in the macroscale without explicit reference to the material constitution or to geometric features of the underlying microstructure. In contrast, the proposed theory relies strictly on homogenization of extensive quantities and derives the macroscopic constitutive response explicitly from the microstructure. A key further novelty of the proposed analysis is in the kinematics of the macroscale, which is naturally enriched by an angular velocity quantifying the local rotatory effect of the motion and enables the decomposition of the kinetic energy into translational and rotational components. The angular velocity is related to a macroscopic quantity akin to a local moment of inertia, whose evolution is governed by its own balance equation. The concept of local moment of inertia in a polar medium was considered initially in [14], where a balance equation is proposed without, however, an associated moment of inertia flux term. Other theories of polar media either neglect the moment of inertia or assume it to be independent of time $[15,16,17]$. In contrast, the present theory provides an explicit definition of a local macroscopic moment of inertia in terms of the microscopic state and a corresponding balance law for its evolution that contains a moment-of-inertia flux term.

The paper also addresses the question of invariance in the macroscale based, again, on a minimal set of assumptions on the form-invariance of the extensive relations and the underlying microscopic balances. Form-invariance of the macroscale balance laws is shown to hold without any extraneous limitations on the nature of the superposed rigid motion. Also, the inertial effects on stress and heat flux are shown to play a crucial role in the transformation of these quantities under superposed rigid-body motions and the associated form-invariance of the balance of linear momentum and energy. In addition, they may point to a path toward the formal resolution of related long-standing controversies on the invariance of stress in turbulence $[18,19,20]$ and heat flux in rotating particle flows $[21,22,23]$.

The organization of the paper is as follows: Section 2 contains an outline of the continuum IrvingKirkwood procedure, as well as expanded discussion on angular momentum. The homogenization of total internal energy and its various constituent parts is addressed in 3 , while the matter invariance is investigated in Section 4 for the principal extensive quantities, Section 5 for stress and linear momentum balance, Section 6 for angular momentum, and Section 7 for heat flux and energy balance. Concluding remarks are offered in Section 8. 


\section{Overview of the Extensive Homogenization Method}

\subsection{Review of previous results: balance of mass and linear momentum}

Consider a body $\mathscr{B}$, which occupies a region $\mathcal{R}$ with boundary $\partial \mathcal{R}$ in the current configuration, and let the positions of material points in the microscale and macroscale be denoted $\mathbf{x}$ and $\mathbf{y}$, respectively. Assuming that continuum mechanics is applicable at both length/time scales, the local forms of the balance laws for mass and linear momentum at the microscale may be expressed as

$$
\begin{gathered}
\dot{\rho}^{m}+\rho^{m} \frac{\partial}{\partial \mathbf{x}} \cdot \mathbf{v}^{m}=0, \\
\rho^{m} \dot{\mathbf{v}}^{m}=\frac{\partial}{\partial \mathbf{x}} \cdot \mathbf{T}^{m}+\rho^{m} \mathbf{b}^{m} .
\end{gathered}
$$

Likewise, the corresponding balance laws for the macroscale take the form

$$
\begin{gathered}
\dot{\rho}^{M}+\rho^{M} \frac{\partial}{\partial \mathbf{y}} \cdot \mathbf{v}^{M}=0, \\
\rho^{M} \dot{\mathbf{v}}^{M}=\frac{\partial}{\partial \mathbf{y}} \cdot \mathbf{T}^{M}+\rho^{M} \mathbf{b}^{M} .
\end{gathered}
$$

Here, $\rho$ is the mass density, $\mathbf{v}$ is the velocity, $\mathbf{T}$ is the Cauchy stress tensor, and $\mathbf{b}$ is the body force per unit mass. In addition, " $\frac{\partial}{\partial \mathbf{y}} . "$ and " $\frac{\partial}{\partial \mathbf{x}}$." denote the divergence operators relative to $\mathbf{y}$ and $\mathbf{x}$, respectively, while the overdot denotes material time derivative. All terms in (1-4) carry a superscript " $m$ " (for microscale) or " $M$ " (for macroscale). Moreover, microscopic terms are functions of $(\mathbf{x}, t)$, while macroscopic terms are functions of $(\mathbf{y}, t)$. For brevity, explicit declaration of these functional dependencies is selectively omitted henceforth.

Expressions for the macroscopic Cauchy stress and body force are derived by postulating homogenization relations for the extensive quantities of mass and linear momentum. These are given by

$$
\begin{aligned}
\rho^{M}(\mathbf{y}, t) & =\int_{\mathcal{R}} \rho^{m}(\mathbf{x}, t) g(\mathbf{y}, \mathbf{x}) \mathrm{d} v^{m}, \\
\rho^{M}(\mathbf{y}, t) \mathbf{v}^{M}(\mathbf{y}, t) & =\int_{\mathcal{R}} \rho^{m}(\mathbf{x}, t) \mathbf{v}^{m}(\mathbf{x}, t) g(\mathbf{y}, \mathbf{x}) \mathrm{d} v^{m},
\end{aligned}
$$

respectively, where $g(\mathbf{y}, \mathbf{x})$ is a real-valued coarse-graining function [2]. This function is assumed to satisfy the condition

$$
g(\mathbf{y}, \mathbf{x})=0 \quad \text { when } \mathbf{x} \in \partial \mathcal{R}
$$

and also be invariant under superposed rigid-body motions, which implies that

$$
g(\mathbf{y}, \mathbf{x})=g\left(\mathbf{y}^{+}, \mathbf{x}^{+}\right) .
$$


The latter has been shown in [2] to further imply that $g(\mathbf{y}, \mathbf{x})=\bar{g}(|\mathbf{x}-\mathbf{y}|)$, hence

$$
\frac{\partial}{\partial \mathbf{x}} g(\mathbf{y}, \mathbf{x})=-\frac{\partial}{\partial \mathbf{y}} g(\mathbf{y}, \mathbf{x})
$$

The support of the coarse-graining function quantifies the length scale which characterizes the homogenization and is informed by the geometry and material constitution of the microstructure.

Taking material time derivatives of relations $(5,6)$, using the balance laws $(1,2)$ at the microscale, and comparing the resulting equations to the balance laws $(3,4)$ at the macroscale, the macroscopic Cauchy stress tensor is found in [2] to be

$$
\mathbf{T}^{M}=\int_{\mathcal{R}}\left[\mathbf{T}^{m}-\rho^{m}\left(\mathbf{v}^{m}-\mathbf{v}^{M}\right) \otimes\left(\mathbf{v}^{m}-\mathbf{v}^{M}\right)\right] g \mathrm{~d} v^{m}
$$

to within a divergence-free term, while the macroscopic body force is given by

$$
\rho^{M} \mathbf{b}^{M}=\int_{\mathcal{R}} \rho^{m} \mathbf{b}^{m} g \mathrm{~d} v^{m}
$$

Equation (10) implies that the macroscopic Cauchy stress is symmetric, as is (on satisfying microscopic angular momentum balance) the corresponding microscopic stress. It also demonstrates the explicit presence of kinetic effects in addition to the (weighted) average of the microscopic stress.

\subsection{Homogenization of angular momentum}

The balance of angular momentum at the macroscale is not considered in the theory originally proposed in [2]. In this section, the consequences of the homogenization of angular momentum are investigated. In particular, the balance of macroscopic angular momentum is derived from its microscopic counterpart, and the associated couple stress tensor is identified along with the body couple in terms of microscopic variables. This process demonstrates the polar nature of the continuum homogenization theory proposed in [2].

Since angular momentum is also an extensive quantity, an additional assumption in the continuous Irving-Kirkwood homogenization theory is that the total macroscopic angular momentum $\mathbf{L}^{M}$ per unit mass is defined as

$$
\rho^{M}(\mathbf{y}, t) \mathbf{L}^{M}(\mathbf{y}, t)=\int_{\mathcal{R}} \mathbf{x} \times \rho^{m}(\mathbf{x}, t) \mathbf{v}^{m}(\mathbf{x}, t) g(\mathbf{y}, \mathbf{x}) \mathrm{d} v^{m} .
$$

This can be alternatively expressed with the aid of (6) as

$$
\rho^{M} \mathbf{L}^{M}=\mathbf{y} \times \rho^{M} \mathbf{v}^{M}+\rho^{M} \mathbf{L}_{s}^{M}
$$


where

$$
\rho^{M} \mathbf{L}_{s}^{M}(\mathbf{y}, t)=\int_{\mathcal{R}}(\mathbf{x}-\mathbf{y}) \times \rho^{m}(\mathbf{x}, t) \mathbf{v}^{m}(\mathbf{x}, t) g(\mathbf{y}, \mathbf{x}) \mathrm{d} v^{m} .
$$

It is readily concluded from (13) that the total macroscopic angular momentum is equal to the macroscopic moment of momentum $\rho^{M} \mathbf{y} \times \mathbf{v}^{M}$ plus the term $\rho^{M} \mathbf{L}_{s}^{M}$ in (14), which is due to the internal spin in the macroscale, see also [11].

The integral form of angular momentum balance in the macroscale may now be expressed as

$$
\frac{\mathrm{d}}{\mathrm{d} t} \int_{\mathcal{P}} \rho^{M} \mathbf{L}^{M} \mathrm{~d} v^{M}=\int_{\mathcal{P}} \mathbf{y} \times \rho^{M} \mathbf{b}^{M} \mathrm{~d} v^{M}+\int_{\partial \mathcal{P}} \mathbf{y} \times \mathbf{t}^{M} \mathrm{~d} a^{M}+\int_{\mathcal{P}} \rho^{M} \mathbf{g}^{M} \mathrm{~d} v^{M}+\int_{\partial \mathcal{P}} \mathbf{m}^{M} \mathrm{~d} a^{M},
$$

where $\mathbf{t}^{M}$ and $\mathbf{m}^{M}$ denote the macroscopic force and force couple on the boundary $\partial \mathcal{P}$ of an arbitrary region $\mathcal{P}$, respectively, while $\mathbf{g}^{M}$ is the body couple in $\mathcal{P}$. Substituting (13) into (15), applying the Reynolds transport and divergence theorems, and invoking $(3,4)$ and the symmetry of the Cauchy stress in (10), the statement of macroscopic angular momentum balance reduces to

$$
\frac{\mathrm{d}}{\mathrm{d} t} \int_{\mathcal{P}} \rho^{M} \mathbf{L}_{s}^{M} \mathrm{~d} v^{M}=\int_{\mathcal{P}} \rho^{M} \mathbf{g}^{M} \mathrm{~d} v^{M}+\int_{\mathcal{P}} \frac{\partial}{\partial \mathbf{y}} \cdot \mathbf{M}^{M} \mathrm{~d} v^{M}
$$

where $\mathbf{M}^{M}$ is the couple stress related to the force couple $\mathbf{m}^{M}$ by the standard Cauchy stress theorem. Equation (16) may be thought of as expressing the balance of the (homogenized) internal spin in the macroscale. A local macroscopic counterpart of (16), derived directly from the Reynolds transport theorem and (3), takes the form

$$
\rho^{M} \dot{\mathbf{L}}_{s}^{M}=\rho^{M} \mathbf{g}^{M}+\frac{\partial}{\partial \mathbf{y}} \cdot \mathbf{M}^{M} .
$$

Expanding the left-hand side of (16) by employing the Reynolds transport theorem and taking advantage of (1), (2), (5-7), (9), the definition in (14), and the symmetry of the microscopic Cauchy stress gives rise to

$$
\begin{aligned}
& \frac{\mathrm{d}}{\mathrm{d} t} \int_{\mathcal{P}} \rho^{M} \mathbf{L}_{s}^{M} \mathrm{~d} v^{M}=\int_{\mathcal{P}}\left[\int_{\mathcal{R}}(\mathbf{x}-\mathbf{y}) \times \rho^{m} \mathbf{b}^{m} g \mathrm{~d} v^{m}\right. \\
& \left.\quad+\frac{\partial}{\partial \mathbf{y}} \cdot \int_{\mathcal{R}}(\mathbf{x}-\mathbf{y}) \times \mathbf{T}^{m} g \mathrm{~d} v^{m}-\frac{\partial}{\partial \mathbf{y}} \cdot \int_{\mathcal{R}}(\mathbf{x}-\mathbf{y}) \times\left[\rho^{m} \mathbf{v}^{m} \otimes\left(\mathbf{v}^{m}-\mathbf{v}^{M}\right)\right] g \mathrm{~d} v^{m}\right] \mathrm{d} v^{M},
\end{aligned}
$$

where the (left) cross product between a vector and a second-order tensor (see, e.g., [24, Section 2.1.7]) is employed in the last two terms of (18). Reconciling the right-hand sides of (16) and (18), it follows that the macroscopic body couple takes the form

$$
\rho^{M} \mathbf{g}^{M}=\int_{\mathcal{R}}(\mathbf{x}-\mathbf{y}) \times \rho^{m} \mathbf{b}^{m} g \mathrm{~d} v^{m},
$$


while, to within a divergence-free term, the macroscopic couple stress is given by

$$
\mathbf{M}^{M}=\int_{\mathcal{R}}(\mathbf{x}-\mathbf{y}) \times \mathbf{T}^{m} g \mathrm{~d} v^{m}-\int_{\mathcal{R}}(\mathbf{x}-\mathbf{y}) \times\left[\rho^{m} \mathbf{v}^{m} \otimes\left(\mathbf{v}^{m}-\mathbf{v}^{M}\right)\right] g \mathrm{~d} v^{m} .
$$

The term on the right-hand side of (19) is due to the internal torque induced by the microscopic body force. Likewise, the two terms comprising the (unsymmetric) macroscopic couple stress in (20) signify the moment of the microscopic stress and the fluctuation in the internal spin, respectively.

It is important to emphasize at this point that the macroscopic angular momentum balance equations do not represent new physics, but rather underline the polar nature of the homogenized macroscopic medium derived from a conventional microscopic continuum. Also, the macroscopic linear momentum balance equations (4) and angular momentum balance equations (17) are coupled by virtue of their dependence on the kinematics and stresses of the (shared) microstructure.

\section{Homogenization of Energy}

In view of the polar nature of the macroscopic continuum, the homogenization of energy in [2] is reconsidered and alternative expressions are derived for the heat supply and heat flux by identifying the appropriate forms of the work by couple stress and body couple. In addition, an additive decomposition of the total internal energy is deduced by a suitable definition of the angular velocity of the macroscopic continuum.

The local form of energy balance in the microscale may be expressed conventionally as

$$
\rho^{m} \dot{e}^{m}=\rho^{m} \mathbf{b}^{m} \cdot \mathbf{v}^{m}+\rho^{m} r^{m}+\frac{\partial}{\partial \mathbf{x}} \cdot\left(\mathbf{T}^{m} \mathbf{v}^{m}\right)-\frac{\partial}{\partial \mathbf{x}} \cdot \mathbf{q}^{m}
$$

where (upon omitting explicit reference to the superscript " $m$ ") $e=\epsilon+\frac{1}{2} \mathbf{v} \cdot \mathbf{v}$ is the total internal energy (including kinetic energy) per unit mass, with $\epsilon$ being the internal energy per unit mass, $\mathbf{q}$ is the heat-flux vector, and $r$ is the heat supply per unit mass. Also, the symmetry of the microscopic Cauchy stress has been invoked in deriving the third term on the right-hand side of (21). The standard reduced form of energy balance in the microscale can be stated as

$$
\rho^{m} \dot{\epsilon}^{m}=\rho^{m} r^{m}+\mathbf{T}^{m} \cdot \frac{\partial \mathbf{v}^{m}}{\partial \mathbf{x}}-\frac{\partial}{\partial \mathbf{x}} \cdot \mathbf{q}^{m} .
$$

It is tempting to put forth an expression for the macroscopic energy balance corresponding to (21), as done previously in [2]. Instead, appreciating the polar nature of the macroscopic continuum, as demonstrated in Section 2.2, it is instructive to start from the statement of extensivity for the total internal energy, in the form

$$
\rho^{M}(\mathbf{y}, t) e^{M}(\mathbf{y}, t)=\int_{\mathcal{R}} \rho^{m}(\mathbf{x}, t) e^{m}(\mathbf{x}, t) g(\mathbf{y}, \mathbf{x}) \mathrm{d} v^{m}
$$


and explore the full range of its implications in relation to macroscopic energy balance. To this end, upon invoking $(5,6)$ and the preceding decomposition of the total microscopic internal energy, equation (23) readily leads to

$$
\rho^{M} e^{M}=\int_{\mathcal{R}} \rho^{m} \epsilon^{m} g \mathrm{~d} v^{m}+\int_{\mathcal{R}} \frac{1}{2} \rho^{m}\left(\mathbf{v}^{m}-\mathbf{v}^{M}\right) \cdot\left(\mathbf{v}^{m}-\mathbf{v}^{M}\right) g \mathrm{~d} v^{m}+\frac{1}{2} \rho^{M} \mathbf{v}^{M} \cdot \mathbf{v}^{M} .
$$

Equation (24) shows that the total macroscopic internal energy consists of three distinct parts: the homogenized microscopic internal energy; the homogenized kinetic energy of the velocity fluctuations in the microscale; and, the macroscopic translational kinetic energy.

To reveal the central role of spin in the macroscopic energy, let $\mathbf{w}^{M}$ be an angular velocity anchored at $\mathbf{y}$, and defer its exact prescription until later in this section. Next, define the convected microscopic velocity $\hat{\mathbf{v}}^{m}$ as

$$
\hat{\mathbf{v}}^{m}=\mathbf{v}^{M}+\mathbf{w}^{M} \times(\mathbf{x}-\mathbf{y}),
$$

where, in general, $\hat{\mathbf{v}}^{m} \neq \mathbf{v}^{m}$, see Figure 1. It is now possible to write the kinetic energy of the fluctuations in $(24)$ as

$$
\begin{aligned}
& \int_{\mathcal{R}} \frac{1}{2} \rho^{m}\left(\mathbf{v}^{m}-\mathbf{v}^{M}\right) \cdot\left(\mathbf{v}^{m}-\mathbf{v}^{M}\right) g \mathrm{~d} v^{m}=\int_{\mathcal{R}} \frac{1}{2} \rho^{m}\left(\mathbf{v}^{m}-\hat{\mathbf{v}}^{m}\right) \cdot\left(\mathbf{v}^{m}-\hat{\mathbf{v}}^{m}\right) g \mathrm{~d} v^{m} \\
& +\int_{\mathcal{R}} \frac{1}{2} \rho^{m}\left[\mathbf{w}^{M} \times(\mathbf{x}-\mathbf{y})\right] \cdot\left[\mathbf{w}^{M} \times(\mathbf{x}-\mathbf{y})\right] g \mathrm{~d} v^{m}+\int_{\mathcal{R}} \rho^{m}\left(\mathbf{v}^{m}-\hat{\mathbf{v}}^{m}\right) \cdot\left[\mathbf{w}^{M} \times(\mathbf{x}-\mathbf{y})\right] g \mathrm{~d} v^{m} .
\end{aligned}
$$

The second term on the right-hand side of (26) can be also expressed as

$$
\begin{aligned}
& \int_{\mathcal{R}} \frac{1}{2} \rho^{m}\left[\mathbf{w}^{M} \times(\mathbf{x}-\mathbf{y})\right] \cdot\left[\mathbf{w}^{M} \times(\mathbf{x}-\mathbf{y})\right] g \mathrm{~d} v^{m} \\
& =\frac{1}{2} \int_{\mathcal{R}} \rho^{m}[(\mathbf{x}-\mathbf{y}) \cdot(\mathbf{x}-\mathbf{y}) \mathbf{i}-(\mathbf{x}-\mathbf{y}) \otimes(\mathbf{x}-\mathbf{y})] g \mathrm{~d} v^{m} \cdot\left(\mathbf{w}^{M} \otimes \mathbf{w}^{M}\right)=\frac{1}{2} \mathbf{I}^{M} \mathbf{w}^{M} \cdot \mathbf{w}^{M},
\end{aligned}
$$

in terms of the (homogenized) moment-of-inertia tensor $\mathbf{I}^{M}$ at point $\mathbf{y}$, defined classically as

$$
\mathbf{I}^{M}=\int_{\mathcal{R}} \rho^{m}[(\mathbf{x}-\mathbf{y}) \cdot(\mathbf{x}-\mathbf{y}) \mathbf{i}-(\mathbf{x}-\mathbf{y}) \otimes(\mathbf{x}-\mathbf{y})] g \mathrm{~d} v^{m},
$$

where $\mathbf{i}$ is the spatial second-order identity tensor.

Starting from (28), it can be readily confirmed with the aid of the Reynolds transport theorem, as well as equations (1), (7), and (9) that

$$
\dot{\mathbf{I}}^{M}+\mathbf{I}^{M} \frac{\partial}{\partial \mathbf{y}} \cdot \mathbf{v}^{M}+\frac{\partial}{\partial \mathbf{y}} \cdot \mathbf{J}^{M}=\mathbf{0},
$$

where

$$
\mathbf{J}^{M}=\int_{\mathcal{R}} \rho^{m}[(\mathbf{x}-\mathbf{y}) \cdot(\mathbf{x}-\mathbf{y}) \mathbf{i}-(\mathbf{x}-\mathbf{y}) \otimes(\mathbf{x}-\mathbf{y})] \otimes\left(\mathbf{v}^{m}-\mathbf{v}^{M}\right) g \mathrm{~d} v^{m}
$$


is a third-order macroscopic moment-of-inertia flux tensor. Equation (29) expresses the (derivable rather than primitive) balance of the moment of inertia and stands in qualitative contrast to the conventional macroscopic mass balance equation (3). In particular, it demonstrates that there is non-material transport of rotational inertia owing to the fluctuations in the velocity, as evidenced by the third term on the left-hand side of (29).

The last term on the right-hand side of (26) can be written with the aid of (25) and (28) as

$$
\int_{\mathcal{R}} \rho^{m}\left(\mathbf{v}^{m}-\hat{\mathbf{v}}^{m}\right) \cdot\left[\mathbf{w}^{M} \times(\mathbf{x}-\mathbf{y})\right] g \mathrm{~d} v^{m}=\int_{\mathcal{R}} \rho^{m}(\mathbf{x}-\mathbf{y}) \times\left(\mathbf{v}^{m}-\mathbf{v}^{M}\right) g \mathrm{~d} v^{m} \cdot \mathbf{w}^{M}-\mathbf{I}^{M} \mathbf{w}^{M} \cdot \mathbf{w}^{M} .
$$

Note that the first term on the right-hand side of (31) involves the spin angular momentum in (14), only considered relative to the macroscopic velocity $\mathbf{v}^{M}$. It is now possible to define the angular velocity $\mathbf{w}^{M}$ such that the left-hand side of (31) vanish identically, which would imply that

$$
\int_{\mathcal{R}} \rho^{m}(\mathbf{x}-\mathbf{y}) \times\left(\mathbf{v}^{m}-\mathbf{v}^{M}\right) g \mathrm{~d} v^{m}=\mathbf{I}^{M} \mathbf{w}^{M} .
$$

Therefore, $\mathbf{w}^{M}$ may be thought of as the angular velocity at $\mathbf{y}$ which, when pre-multiplied by the (local) moment-of-inertia tensor, quantifies the internal spin relative to the macroscopic velocity.

The preceding definition effectively eliminates the coupling between the translational and rotational velocity in the internal energy of (24). Indeed, taking into account equations (26), (27), and (31), in connection with the definition of $\mathbf{w}^{M}$ in (32), the total macroscopic internal energy in (24) now takes the additive form

$$
\rho^{M} e^{M}=\rho^{M} \epsilon^{M}+\frac{1}{2} \rho^{M} \mathbf{v}^{M} \cdot \mathbf{v}^{M}+\frac{1}{2} \mathbf{I}^{M} \mathbf{w}^{M} \cdot \mathbf{w}^{M},
$$

where the macroscopic internal energy $\epsilon^{M}$ per unit mass is defined as

$$
\rho^{M} \epsilon^{M}=\int_{\mathcal{R}} \rho^{m} \epsilon^{m} g \mathrm{~d} v^{m}+\int_{\mathcal{R}} \frac{1}{2} \rho^{m}\left(\mathbf{v}^{m}-\hat{\mathbf{v}}^{m}\right) \cdot\left(\mathbf{v}^{m}-\hat{\mathbf{v}}^{m}\right) g \mathrm{~d} v^{m} .
$$

The last two terms in (33) correspond respectively to the macroscopic kinetic energy due to translational and rotational effects. Moreover, $\rho^{M} \epsilon^{M}$ in (34) is the macroscopic internal energy due to all sources other than (macroscopic) kinetic energy and includes the effect of kinetic energy fluctuations relative to the convected velocity $\hat{\mathbf{v}}^{m}$, which are understood here as a manifestation of thermal, rather than mechanical, energy.

Starting from the extensivity relation (23), a local statement of macroscopic energy balance may be derived (see Appendix A) in the form

$$
\rho^{M} \dot{e}^{M}=\rho^{M} \mathbf{b}^{M} \cdot \mathbf{v}^{M}+\rho^{M} \mathbf{g}^{M} \cdot \mathbf{w}^{M}+\int_{\mathcal{R}}\left[\rho^{m} r^{m}+\rho^{m} \mathbf{b}^{m} \cdot\left(\mathbf{v}^{m}-\hat{\mathbf{v}}^{m}\right)\right] g \mathrm{~d} v^{m}+
$$




$$
\frac{\partial}{\partial \mathbf{y}} \cdot\left(\mathbf{T}^{M} \mathbf{v}^{M}\right)+\frac{\partial}{\partial \mathbf{y}} \cdot\left[\left(\mathbf{M}^{M}\right)^{T} \mathbf{w}^{M}\right]-\frac{\partial}{\partial \mathbf{y}} \cdot \int_{\mathcal{R}}\left[\mathbf{q}^{m}-\mathbf{T}^{m}\left(\mathbf{v}^{m}-\hat{\mathbf{v}}^{m}\right)+\rho^{m} \hat{e}^{m}\left(\mathbf{v}^{m}-\mathbf{v}^{M}\right)\right] g \mathrm{~d} v^{m}
$$

where

$$
\hat{e}^{m}=\epsilon^{m}+\frac{1}{2}\left(\mathbf{v}^{m}-\hat{\mathbf{v}}^{m}\right) \cdot\left(\mathbf{v}^{m}-\hat{\mathbf{v}}^{m}\right)-\frac{1}{2} \hat{\mathbf{v}}^{m} \cdot \hat{\mathbf{v}}^{m},
$$

and the superscript " $T$ " signifies tensorial transpose. In contrast to its microscopic counterpart in (21), equation (35) contains power terms involving the body couple $\mathbf{g}^{M}$ and the couple stress $\mathbf{M}^{M}$, thus further demonstrating the polar nature of the homogenized macroscopic continuum. Also, as seen directly from (36), the quantity $\hat{e}^{m}$ comprises two competing energetic contributions: first, the microscopic internal energy including the kinetic energy of the fluctuations of the microscopic velocity relative to the "rigid-body motion" induced (locally) by $\mathbf{v}^{M}$ and $\mathbf{w}^{M}$, and second, the kinetic energy of the same motion.

The structure of equation (35) implies that the macroscopic heat supply may be defined as

$$
\rho^{M} r^{M}=\int_{\mathcal{R}}\left[\rho^{m} r^{m}+\rho^{m} \mathbf{b}^{m} \cdot\left(\mathbf{v}^{m}-\hat{\mathbf{v}}^{m}\right)\right] g \mathrm{~d} v^{m},
$$

while, to within a divergence-free term, the macroscopic heat flux is given by

$$
\mathbf{q}^{M}=\int_{\mathcal{R}}\left[\mathbf{q}^{m}-\mathbf{T}^{m}\left(\mathbf{v}^{m}-\hat{\mathbf{v}}^{m}\right)+\rho^{m} \hat{e}^{m}\left(\mathbf{v}^{m}-\mathbf{v}^{M}\right)\right] g \mathrm{~d} v^{m} .
$$

With the preceding definitions in place, the local statement of macroscopic energy balance (35) takes the form

$$
\rho^{M} \dot{e}^{M}=\rho^{M} \mathbf{b}^{M} \cdot \mathbf{v}^{M}+\rho^{M} \mathbf{g}^{M} \cdot \mathbf{w}^{M}+\rho^{M} r^{M}+\frac{\partial}{\partial \mathbf{y}} \cdot\left(\mathbf{T}^{M} \mathbf{v}^{M}\right)+\frac{\partial}{\partial \mathbf{y}} \cdot\left[\left(\mathbf{M}^{M}\right)^{T} \mathbf{w}^{M}\right]-\frac{\partial}{\partial \mathbf{y}} \cdot \mathbf{q}^{M}
$$

It is important to observe here that the definitions in $(37,38)$ and the energy balance statement in (39) readily reduce to those derived in [2] upon neglecting the angular velocity $\mathbf{w}^{M}$, which is tantamount to outright suppressing the polar effects in the macroscale.

\section{Invariance of Extensive Relations}

In this section, the question of invariance under superposed rigid-body motions is investigated systematically for the relations (5), (6), (12), and (23) between the principal extensive quantities in the two scales.

By way of background, recall that, under superposed rigid-body motions, any macroscopic point $\mathbf{y}$ in the current configuration of $\mathcal{R}$ is mapped to

$$
\mathbf{y}^{+}=\mathrm{Qy}+\mathbf{c}
$$


where $\mathbf{Q}$ is an arbitrary time-dependent rotation tensor and $\mathbf{c}$ is an arbitrary time-dependent translation vector. It follows from (40) that the corresponding velocity and acceleration of this point are given by

$$
\begin{gathered}
\mathbf{v}^{M^{+}}=\mathbf{Q} \mathbf{v}^{M}+\dot{\mathbf{Q}} \mathbf{y}+\dot{\mathbf{c}} \\
\dot{\mathbf{v}}^{M^{+}}=\mathbf{Q} \dot{\mathbf{v}}^{M}+2 \dot{\mathbf{Q}} \mathbf{v}^{M}+\ddot{\mathbf{Q}} \mathbf{y}+\ddot{\mathbf{c}}
\end{gathered}
$$

respectively. In complete analogy to (40-42), one may express the position, velocity and acceleration of any microscopic material point $\mathbf{x}$ under the same superposed rigid-body motion as

$$
\begin{gathered}
\mathbf{x}^{+}=\mathbf{Q} \mathbf{x}+\mathbf{c}, \\
\mathbf{v}^{m+}=\mathbf{Q} \mathbf{v}^{m}+\dot{\mathbf{Q}} \mathbf{x}+\dot{\mathbf{c}}, \\
\dot{\mathbf{v}}^{m+}=\mathbf{Q} \dot{\mathbf{v}}^{m}+2 \dot{\mathbf{Q}} \mathbf{v}^{m}+\ddot{\mathbf{Q}} \mathbf{x}+\ddot{\mathbf{c}},
\end{gathered}
$$

respectively.

At this stage, it is postulated that the relations (5), (6), (13), and (23) which connect the two scales must be form-invariant under superposed rigid-body motions (in the sense of $[25,26]$ ). This reflects the physically plausible idea that extensive quantities should remain extensive under superposed rigid-body motion. Furthermore, it is assumed that the microscopic balance laws in (1), (2), (21) are likewise form-invariant under superposed rigid-body motions.

Starting with (5), form-invariance implies that

$$
\rho^{M^{+}}\left(\mathbf{y}^{+}, t\right)=\int_{\mathcal{R}^{+}} \rho^{m+}\left(\mathbf{x}^{+}, t\right) g\left(\mathbf{y}^{+}, \mathbf{x}^{+}\right) \mathrm{d} v^{m+} .
$$

Upon recalling that $\rho^{m+}=\rho^{m}$ is necessary to ensure form-invariance of the microscopic mass balance, noting that volume are unchanged under superposed rigid-body motions in the microscale (that is, $\mathrm{d} v^{m+}=\mathrm{d} v^{m}$ ), and also using (8), it follows immediately from (46) that

$$
\rho^{M^{+}}=\rho^{M} .
$$

Form-invariance of the extensive relation (6) for linear momentum necessitates that

$$
\rho^{M^{+}}\left(\mathbf{y}^{+}, t\right) \mathbf{v}^{M^{+}}\left(\mathbf{y}^{+}, t\right)=\int_{\mathcal{R}^{+}} \rho^{m+} \mathbf{v}^{m+} g\left(\mathbf{y}^{+}, \mathbf{x}^{+}\right) \mathrm{d} v^{m+} .
$$

Substituting the expressions for the macroscopic and microscopic velocities from (41) and (44), appealing to (6), and using, again, (8) and the invariance of density and infinitesimal volume in the microscale, equation (48) yields

$$
\dot{\mathbf{Q}}\left(\rho^{M} \mathbf{y}-\int_{\mathcal{R}} \rho^{m} \mathbf{x} g \mathrm{~d} v^{m}\right)+\dot{\mathbf{c}}\left(\rho^{M}-\int_{\mathcal{R}} \rho^{m} g \mathrm{~d} v^{m}\right)=\mathbf{0} .
$$


Setting $\dot{\mathbf{Q}}=\mathbf{0}$ in (49), it follows from the arbitrariness of $\dot{\mathbf{c}}$ that the homogenization relation (5) for mass may be derived (rather than assumed at the outset) from the invariance of the homogenization relation (6) for linear momentum. Moreover, upon defining the skew-symmetric tensor $\boldsymbol{\Omega}=\mathbf{Q}^{T} \mathbf{\mathbf { Q }}$ and its associated axial vector $\boldsymbol{\omega}$, it follows from the reduced form of (49) and the arbitrariness of $\mathbf{Q}$ that

$$
\boldsymbol{\omega} \times\left(\rho^{M} \mathbf{y}-\int_{\mathcal{R}} \rho^{m} \mathbf{x} g(\mathbf{y}, \mathbf{x}) \mathrm{d} v^{m}\right)=\mathbf{0} .
$$

Since (50) is valid for all vectors $\boldsymbol{\omega}$, it is concluded that

$$
\rho^{M} \mathbf{y}=\int_{\mathcal{R}} \rho^{m} \mathbf{x} g(\mathbf{y}, \mathbf{x}) \mathrm{d} v^{m}
$$

which necessitates that the macroscopic point $\mathbf{y}$ be located at the ( $g$-weighted) center of mass of the microscopic region around $\mathbf{y}$. Furthermore, starting from (51), it can be readily shown with the aid of (40), (43), (47), together with (5), (8), and the invariance of microscopic density and volume that

$$
\rho^{M^{+}} \mathbf{y}^{+}=\int_{\mathcal{R}^{+}} \rho^{m+} \mathbf{x}^{+} g\left(\mathbf{y}^{+}, \mathbf{x}^{+}\right) \mathrm{d} v^{m+},
$$

therefore the center-of-mass condition (51) is itself form-invariant. This condition is of practical importance in computations, where its violation would lead to compounding errors, a point which is already well-recognized in the related molecular dynamics literature [27]. An immediate implication of (51) is that the spin angular momentum in (14) now coincides with its counterpart relative to the center of mass, which enters the definition of the angular velocity $\mathbf{w}^{M}$ in (32). A further implication of (51), in conjunction with (30) is in restating the macroscopic balance of angular momentum equation (17) in terms of the angular velocity $\mathbf{w}^{M}$ as

$$
\mathbf{I}^{M} \dot{\mathbf{w}}^{M}=\rho^{M} \mathbf{g}^{M}+\frac{\partial}{\partial \mathbf{y}} \cdot \mathbf{M}^{M}+\left(\frac{\partial}{\partial \mathbf{y}} \cdot \mathbf{J}^{M}\right) \mathbf{w}^{M}
$$

with the last term on the right-hand side reflecting, again, the effect of the non-material transport of rotational inertia. Moreover, upon also taking advantage of (34), as well as of (3), (4), (29), and (53), the reduced form of the energy balance equation in the macroscale is easily derived from (39) as

$$
\rho^{M} \dot{\epsilon}^{M}=\rho^{M} r^{M}+\mathbf{T}^{M} \cdot \frac{\partial \mathbf{v}^{M}}{\partial \mathbf{y}}+\mathbf{M}^{M} \cdot \frac{\partial \mathbf{w}^{M}}{\partial \mathbf{y}}-\frac{1}{2}\left(\frac{\partial}{\partial \mathbf{y}} \cdot \mathbf{J}^{M}\right) \mathbf{w}^{M} \cdot \mathbf{w}^{M}-\frac{\partial}{\partial \mathbf{y}} \cdot \mathbf{q}^{M} .
$$

Again, it is instructive to compare (54) to its microscopic counterpart (22).

Proceeding to angular momentum, form-invariance of the extensive relation (13) implies that

$$
\rho^{M^{+}} \mathbf{L}^{M^{+}}=\mathbf{y}^{+} \times \rho^{M^{+}} \mathbf{v}^{M^{+}}+\rho^{M^{+}} \mathbf{L}_{s}^{M^{+}}
$$


This does not yield additional restrictions on any kinematic or kinetic variables. Rather, it furnishes an explicit relation between the angular momenta $\rho^{M} \mathbf{L}^{M}$ and $\rho^{{ }^{+}} \mathbf{L}^{M^{+}}$. To derive this relation, start with the spin angular momentum term in (55) and observe, using (14), (28), (40), (43), (44), (51), as well as the invariance of microscopic density and volume, that

$$
\begin{aligned}
\rho^{M^{+}} \mathbf{L}_{s}^{M^{+}} & =\int_{\mathcal{R}^{+}}\left(\mathbf{x}^{+}-\mathbf{y}^{+}\right) \times \rho^{m+} \mathbf{v}^{m+} g^{+} \mathrm{d} v^{m+} \\
& =\int_{\mathcal{R}}[\mathbf{Q}(\mathbf{x}-\mathbf{y})] \times \rho^{m}\left(\mathbf{Q} \mathbf{v}^{m}+\dot{\mathbf{Q}} \mathbf{x}+\dot{\mathbf{c}}\right) g \mathrm{~d} v^{m} \\
& =\mathbf{Q} \rho^{M} \mathbf{L}_{s}^{M}+\mathbf{Q} \mathbf{I}^{M} \boldsymbol{\omega},
\end{aligned}
$$

where, for brevity, $g^{+}=g\left(\mathbf{y}^{+}, \mathbf{x}^{+}\right)$. The preceding relation shows that the deviation of spin angular momentum from invariance equals an (additive) contribution due to the angular velocity $\boldsymbol{\omega}$ of the superposed rigid-body motion. Substituting (56) into (55) and recalling (40), (41) and (47), it follows that

$$
\begin{aligned}
\rho^{M^{+}} \mathbf{L}^{M^{+}}=\mathbf{Q} \rho^{M} \mathbf{L}^{M}+\mathbf{Q}\left[\rho^{M}[(\mathbf{y} \cdot \mathbf{y}) \mathbf{i}-\mathbf{y} \otimes \mathbf{y}]+\mathbf{I}^{M}\right] \boldsymbol{\omega} & \\
& +(\mathbf{Q} \mathbf{y}+\mathbf{c}) \times \rho^{M} \dot{\mathbf{c}}+\mathbf{c} \times \rho^{M} \mathbf{Q}\left(\mathbf{v}^{M}+\boldsymbol{\omega} \times \mathbf{y}\right) .
\end{aligned}
$$

As seen from (57), additional angular momentum is generated by the superposed angular velocity $\boldsymbol{\omega}$, the superposed translational velocity $\dot{\mathbf{c}}$ and the coupling of the macroscopic velocity with the superposed translation and rotation.

Lastly, imposing form-invariance to the energy relation (23), as further expanded in (33) and (34), leads to

$$
\rho^{M^{+}} e^{M^{+}}=\rho^{M^{+}} \epsilon^{M^{+}}+\frac{1}{2} \rho^{M^{+}} \mathbf{v}^{M^{+}} \cdot \mathbf{v}^{M^{+}}+\frac{1}{2} \mathbf{I}^{M^{+}} \mathbf{w}^{M^{+}} \cdot \mathbf{w}^{M^{+}}
$$

where

$$
\rho^{M^{+}} \epsilon^{M^{+}}=\int_{\mathcal{R}^{+}} \rho^{m+} \epsilon^{m+} g^{+} \mathrm{d} v^{m+}+\int_{\mathcal{R}^{+}} \frac{1}{2} \rho^{m+}\left(\mathbf{v}^{m+}-\hat{\mathbf{v}}^{m+}\right) \cdot\left(\mathbf{v}^{m+}-\hat{\mathbf{v}}^{m+}\right) g^{+} \mathrm{d} v^{m+} .
$$

To start exploring the implications of (58), observe that

$$
\begin{aligned}
\mathbf{I}^{M^{+}} & =\int_{\mathcal{R}^{+}} \rho^{m+}\left[\left(\mathbf{x}^{+}-\mathbf{y}^{+}\right) \cdot\left(\mathbf{x}^{+}-\mathbf{y}^{+}\right) \mathbf{i}-\left(\mathbf{x}^{+}-\mathbf{y}^{+}\right) \otimes\left(\mathbf{x}^{+}-\mathbf{y}^{+}\right)\right] g^{+} \mathrm{d} v^{m+} \\
& =\int_{\mathcal{R}} \rho^{m}[[\mathbf{Q}(\mathbf{x}-\mathbf{y})] \cdot[\mathbf{Q}(\mathbf{x}-\mathbf{y})] \mathbf{i}-[\mathbf{Q}(\mathbf{x}-\mathbf{y})] \otimes[\mathbf{Q}(\mathbf{x}-\mathbf{y})]] g \mathrm{~d} v^{m} \\
& =\mathbf{Q I}^{M} \mathbf{Q}^{T}
\end{aligned}
$$


a well-known result in rigid-body dynamics, which follows from (40), (43), (8), and the invariance of microscopic density and volume. Next, upon taking advantage of the definition (32), written under superposed rigid-body motion as

$$
\int_{\mathcal{R}^{+}} \rho^{m+}\left(\mathbf{x}^{+}-\mathbf{y}^{+}\right) \times\left(\mathbf{v}^{m+}-\mathbf{v}^{M^{+}}\right) g^{+} \mathrm{d} v^{m+}=\mathbf{I}^{M^{+}} \mathbf{w}^{M^{+}},
$$

one may relate the angular velocity $\mathbf{w}^{M}$ to its counterpart $\mathbf{w}^{M^{+}}$. To wit,

$$
\begin{aligned}
\mathbf{I}^{M^{+}} \mathbf{w}^{M^{+}} & =\int_{\mathcal{R}} \rho^{m}[\mathbf{Q}(\mathbf{x}-\mathbf{y})] \times\left[\mathbf{Q}\left(\mathbf{v}^{m}-\mathbf{v}^{M}\right)+\dot{\mathbf{Q}}(\mathbf{x}-\mathbf{y})\right] g \mathrm{~d} v^{m} \\
& =\mathbf{Q} \int_{\mathcal{R}} \rho^{m}(\mathbf{x}-\mathbf{y}) \times\left(\mathbf{v}^{m}-\mathbf{v}^{M}\right) g \mathrm{~d} v^{m}+\mathbf{Q} \int_{\mathcal{R}} \rho^{m}(\mathbf{x}-\mathbf{y}) \times \mathbf{\Omega}(\mathbf{x}-\mathbf{y}) g \mathrm{~d} v^{m} \\
& =\mathbf{Q I}^{M} \mathbf{w}^{M}+\mathbf{Q I}^{M} \boldsymbol{\omega},
\end{aligned}
$$

upon invoking (28) and, once again, (32). This, in conjunction with (60), implies that

$$
\mathbf{w}^{M^{+}}=\mathbf{Q}\left(\mathbf{w}^{M}+\boldsymbol{\omega}\right)
$$

which reveals the additive effect of the superposed angular velocity on $\mathbf{w}^{M}$. It now follows from (40), (41), (43), (44), and (63) that, under superposed rigid-body motions, the relative velocity $\mathbf{v}^{m}-\hat{\mathbf{v}}^{m}$ transforms as

$$
\begin{aligned}
\mathbf{v}^{m+}-\hat{\mathbf{v}}^{m+} & =\mathbf{v}^{m+}-\mathbf{v}^{M^{+}}-\mathbf{w}^{M^{+}} \times\left(\mathbf{x}^{+}-\mathbf{y}^{+}\right) \\
& =\mathbf{Q}\left(\mathbf{v}^{m}-\mathbf{v}^{M}\right)+\dot{\mathbf{Q}}(\mathbf{x}-\mathbf{y})-\mathbf{Q}\left(\mathbf{w}^{M}+\boldsymbol{\omega}\right) \times \mathbf{Q}(\mathbf{x}-\mathbf{y}) \\
& =\mathbf{Q}\left(\mathbf{v}^{m}-\hat{\mathbf{v}}^{m}\right)
\end{aligned}
$$

which proves that this term (unlike $\mathbf{v}^{m}-\mathbf{v}^{M}$ ) is objective. Next, recalling that the assumed forminvariance of the microscopic energy balance (22) is satisfied provided that

$$
\epsilon^{m+}=\epsilon^{m}, r^{m+}=r^{m}, \quad \mathbf{q}^{m+}=\mathbf{Q q}^{m},
$$

it can be shown starting from (59), with the aid of (64) and (65) 1 , that

$$
\rho^{M^{+}} \epsilon^{M^{+}}=\rho^{M} \epsilon^{M} .
$$

This means that the macroscopic internal energy (including the kinetic energy of the velocity fluctuations relative to $\hat{\mathbf{v}}^{m}$ ) is unaffected by superposed rigid-body motions, a result which is highly desirable on physical grounds. A straightforward calculation shows that the total internal energy in (58) relates to its counterpart before the superposition of a rigid-body motion according to 


$$
\begin{aligned}
\rho^{M^{+}} e^{M^{+}}=\rho^{M} e^{M}+\frac{1}{2} \rho^{M}(\boldsymbol{\omega} \times \mathbf{y}) & \cdot(\boldsymbol{\omega} \times \mathbf{y})+\frac{1}{2} \rho^{M} \dot{\mathbf{c}} \cdot \dot{\mathbf{c}}+\frac{1}{2} \mathbf{I}^{M} \boldsymbol{\omega} \cdot \boldsymbol{\omega} \\
& +\dot{\mathbf{c}} \cdot \rho^{M} \mathbf{Q}\left(\mathbf{v}^{M}+\boldsymbol{\omega} \times \mathbf{y}\right)+\boldsymbol{\omega} \cdot\left(\rho^{M} \mathbf{y} \times \mathbf{v}^{M}+\mathbf{I}^{M} \mathbf{w}^{M}\right),
\end{aligned}
$$

with each of the additional terms on the right-hand side corresponding to contributions due to the superposed rigid translation and rotation.

\section{Invariance: Macroscopic Cauchy Stress and Linear Momentum Balance}

Under a superposed rigid-body motion, the macroscopic Cauchy stress of (10) becomes

$$
\mathbf{T}^{M^{+}}=\int_{\mathcal{R}}\left[\mathbf{T}^{m+}-\rho^{m+}\left(\mathbf{v}^{m+}-\mathbf{v}^{M^{+}}\right) \otimes\left(\mathbf{v}^{m+}-\mathbf{v}^{M^{+}}\right)\right] g^{+} \mathrm{d} v^{m+}
$$

Assuming the usual invariance relation $\mathbf{T}^{m^{+}}=\mathbf{Q} \mathbf{T}^{m} \mathbf{Q}^{T}$ at the microscale, see, e.g., [28], appealing to the invariance of microscopic density and volume, and exploiting (8) and the transformation equations (41) and (44) for the velocity, (68) yields

$$
\begin{aligned}
\mathbf{T}^{M^{+}}= & \int_{\mathcal{R}}\left[\mathbf{Q} \mathbf{T}^{m} \mathbf{Q}^{T}-\rho^{m}\left[\mathbf{Q}\left(\mathbf{v}^{m}-\mathbf{v}^{M}\right)+\dot{\mathbf{Q}}(\mathbf{x}-\mathbf{y})\right] \otimes\left[\mathbf{Q}\left(\mathbf{v}^{m}-\mathbf{v}^{M}\right)+\dot{\mathbf{Q}}(\mathbf{x}-\mathbf{y})\right]\right] g \mathrm{~d} v^{m} \\
= & \mathbf{Q}\left[\int_{\mathcal{R}}\left[\mathbf{T}^{m}-\rho^{m}\left(\mathbf{v}^{m}-\mathbf{v}^{M}\right) \otimes\left(\mathbf{v}^{m}-\mathbf{v}^{M}\right)\right] g \mathrm{~d} v^{m}\right] \mathbf{Q}^{T}-\int_{\mathcal{R}} \rho^{m} \mathbf{Q}\left(\mathbf{v}^{m}-\mathbf{v}^{M}\right) \otimes \dot{\mathbf{Q}}(\mathbf{x}-\mathbf{y}) g \mathrm{~d} v^{m} \\
& -\int_{\mathcal{R}} \rho^{m} \dot{\mathbf{Q}}(\mathbf{x}-\mathbf{y}) \otimes \mathbf{Q}\left(\mathbf{v}^{m}-\mathbf{v}^{M}\right) g \mathrm{~d} v^{m}-\int_{\mathcal{R}} \rho^{m} \dot{\mathbf{Q}}(\mathbf{x}-\mathbf{y}) \otimes \dot{\mathbf{Q}}(\mathbf{x}-\mathbf{y}) g \mathrm{~d} v^{m}
\end{aligned}
$$

Recalling (10) and the definition of $\boldsymbol{\Omega}$, the preceding equation may be rewritten compactly as

$$
\mathbf{T}^{M^{+}}=\mathbf{Q T}^{M} \mathbf{Q}^{T}-\mathbf{Q}\left[\boldsymbol{\Omega A}+(\boldsymbol{\Omega} \mathbf{A})^{T}+\mathbf{\Omega} \mathbf{B} \mathbf{\Omega}^{T}\right] \mathbf{Q}^{T}
$$

where

$$
\mathbf{A}=\int_{\mathcal{R}} \rho^{m}(\mathbf{x}-\mathbf{y}) \otimes\left(\mathbf{v}^{m}-\mathbf{v}^{M}\right) g \mathrm{~d} v^{m}
$$

and

$$
\mathbf{B}=\int_{\mathcal{R}} \rho^{m}(\mathbf{x}-\mathbf{y}) \otimes(\mathbf{x}-\mathbf{y}) g \mathrm{~d} v^{m} .
$$

Note that the tensor $\mathbf{B}$ is symmetric, hence the symmetry of the macroscopic Cauchy stress in (70) is preserved.

It is clear from (70) that the transformation of the macroscopic Cauchy stress under superposed rigid-body motions does not generally obey the conventional continuum mechanics invariance relation. In fact, all of the additional terms on the right-hand side of (70) involve the angular velocity $\boldsymbol{\Omega}$. 
Indeed, the first (symmetrized) pair of terms reflects the contribution of the angular momentum due to the fluctuations $\mathbf{v}^{m}-\mathbf{v}^{M}$, while the last term quantifies the effect of the unit cell's moment of inertia on the macroscopic stress. It is shown in Appendix B that these additional terms are individually divergence-free with respect to the macroscopic coordinates of the system in the rigidly transformed frame, hence they do not affect the balance of linear momentum in that frame. Clearly, if the contribution of the velocity fluctuation terms in (10) is negligible (which would be a reasonable assumption for most problems involving solids), objectivity of the Cauchy stress tensor is restored.

An important additional implication of the divergence-free property of the non-invariant terms in (70) is in the question of form-invariance for the macroscopic linear momentum balance. Indeed, recalling that, in view of (42), form-invariance of the microscopic linear momentum balance translates to the condition

$$
\rho^{m} \mathbf{b}^{m+}=\mathbf{Q} \rho^{m} \mathbf{b}^{m}+\rho^{m}\left(2 \dot{\mathbf{Q}} \mathbf{v}^{m}+\ddot{\mathbf{Q}} \mathbf{x}+\ddot{\mathbf{c}}\right)
$$

one may readily conclude with the aid of (4-6), (11), (42), (45), (47), (51), and (70) that

$$
\rho^{M^{+}} \dot{\mathbf{v}}^{M^{+}}=\frac{\partial}{\partial \mathbf{y}^{+}} \cdot \mathbf{T}^{M^{+}}+\rho^{M^{+}} \mathbf{b}^{M^{+}}
$$

where

$$
\rho^{M^{+}} \mathbf{b}^{M^{+}}=\int_{\mathcal{R}^{+}} \rho^{m+} \mathbf{b}^{m+} g^{+} \mathrm{d} v^{m+}=\mathbf{Q} \rho^{M} \mathbf{b}^{M}+\rho^{M}\left(2 \dot{\mathbf{Q}} \mathbf{v}^{M}+\ddot{\mathbf{Q}} \mathbf{y}+\ddot{\mathbf{c}}\right) .
$$

The importance of the transformation condition (70) is alluded to in $[18,19]$, where it is observed that satisfaction of the conventional invariance requirement by the (macroscopic) stress is tantamount to ignoring the effects of inertia in the constitutive prescription of stress. This observation applies regardless of the question of invariance of the balance laws themselves.

\section{Invariance: Couples and Angular Momentum Balance}

Returning to the macroscopic angular momentum balance equation (17), one may confirm by direct calculation that it is intrinsically (that is, without the need for any additional assumptions) forminvariant. Furthermore, starting from the respective definitions in (19) and (20), it can be shown with the aid of (8), (40), (41), (43), (44), (73), as well as the invariance of microscopic stress, mass density, and volume, that

$$
\begin{aligned}
\rho^{M^{+}} \mathbf{g}^{M^{+}} & =\int_{\mathcal{R}^{+}}\left(\mathbf{x}^{+}-\mathbf{y}^{+}\right) \times \rho^{m+} \mathbf{b}^{m+} g^{+} \mathrm{d} v^{m+} \\
& =\mathbf{Q} \rho^{M} \mathbf{g}^{M}+\mathbf{Q} \int_{\mathcal{R}}(\mathbf{x}-\mathbf{y}) \times \rho^{m}\left(2 \mathbf{\Omega} \mathbf{v}^{m}+\mathbf{Q}^{T} \ddot{\mathbf{Q}} \mathbf{x}\right) g \mathrm{~d} v^{m}
\end{aligned}
$$


and

$$
\begin{aligned}
& \mathbf{M}^{M^{+}}=\int_{\mathcal{R}^{+}}\left(\mathbf{x}^{+}-\mathbf{y}^{+}\right) \times \mathbf{T}^{m+} g^{+} \mathrm{d} v^{m+}-\int_{\mathcal{R}^{+}}\left(\mathbf{x}^{+}-\mathbf{y}^{+}\right) \times\left[\rho^{m+} \mathbf{v}^{m+} \otimes\left(\mathbf{v}^{m+}-\mathbf{v}^{M^{+}}\right)\right] g^{+} \mathrm{d} v^{m+} \\
&=\mathbf{Q M}^{M} \mathbf{Q}^{T}- \\
& \mathbf{Q} \int_{\mathcal{R}}(\mathbf{x}-\mathbf{y}) \times \rho^{m}\left[\left(\boldsymbol{\Omega} \mathbf{x}+\mathbf{Q}^{T} \dot{\mathbf{c}}\right) \otimes\left[\left(\mathbf{v}^{m}-\mathbf{v}^{M}\right)+\boldsymbol{\Omega}(\mathbf{x}-\mathbf{y})\right]+\mathbf{v}^{m} \otimes \boldsymbol{\Omega}(\mathbf{x}-\mathbf{y})\right] g \mathrm{~d} v^{m} \mathbf{Q}^{T} .
\end{aligned}
$$

The preceding two equations demonstrate that neither the body couple nor the couple stress is objective, which is entirely reasonable given their physical meaning. Again, it is easy to show that the couple stress would be objective if the contribution of the velocity fluctuations can be ignored in $(20)$.

\section{Invariance: Macroscopic Heat Flux and Energy Balance}

Under superposed rigid-body motions, the macroscopic heat flux vector in (38) is given by

$$
\mathbf{q}^{M^{+}}=\int_{\mathcal{R}^{+}}\left[\mathbf{q}^{m+}+\mathbf{T}^{m^{+}}\left(\mathbf{v}^{m+}-\hat{\mathbf{v}}^{m+}\right)+\rho^{m+} \hat{e}^{m+}\left(\mathbf{v}^{m+}-\mathbf{v}^{M^{+}}\right)\right] g^{+} \mathrm{d} v^{m+} .
$$

Taking into consideration the invariance properties of the microscopic stress and heat flux, and invoking (8), (38), (40,41), (43,44), and (64), the preceding expression leads to

$$
\mathbf{q}^{M^{+}}=\mathbf{Q} \mathbf{q}^{M}+\mathbf{Q}\left[\boldsymbol{\Omega} \int_{\mathcal{R}} \rho^{m} \hat{e}^{m+}(\mathbf{x}-\mathbf{y}) g \mathrm{~d} v^{m}+\int_{\mathcal{R}} \rho^{m}\left(\hat{e}^{m+}-\hat{e}^{m}\right)\left(\mathbf{v}^{m}-\mathbf{v}^{M}\right) g \mathrm{~d} v^{m}\right] .
$$

As with the Cauchy stress, it is seen from (79) that the macroscopic heat flux is not invariant under superposed rigid-body motions, as previously observed [21, 22, 23]. However, unlike stress, the nonobjective parts of the heat flux in (79) are neither individually nor jointly divergence-free relative to the coordinates in the superposed configuration.

As with the balances of mass, linear momentum, and angular momentum, the macroscopic balance of energy is form-invariant. This can be argued in a straightforward manner by invoking the form-invariance of the total energy in (23) and repeating the derivation of the energy balance equation contained in Appendix B using the superposed configuration while exploiting the forminvariance of the microscopic energy balance (21).

\section{Conclusions}

The continuum-to-continuum homogenization theory inspired by the Irving-Kirkwood procedure gives rise to a polar macroscopic medium due to the length scale inherent in the coarse-graining 
process. The role of macroscopic angular momentum becomes non-trivial and a suitable definition of the local macroscopic spin enables the additive decomposition of the total internal energy into non-inertial, translational and rotational components, thus enabling a canonical representation of the contributions of internal forces and stresses (both polar and non-polar) in the macroscopic balance of energy.

The assumption of form-invariance of the extensive relations for mass, linear and angular momenta, and total energy combined with the standard invariance properties in the microscale suffices in translating the form-invariance of the microscopic balance laws to the macroscale, thereby providing a sound theoretical foundation for future development of macroscopic models. At the same time, the homogenization theory yields macroscopic stresses and heat fluxes that do not observe the conventional invariance requirement due to presence of inertial effects. These departures, which have been long observed in fluctuation-dominated problems, such as turbulent flows, are now placed within the realm of a continuum-mechanical theory.

In broader terms, the paper demonstrates that continuum-to-continuum homogenization may be an effective vehicle for investigating (and, hopefully, expanding) the boundaries of traditional continuum-mechanics, as motivated by the study of inhomogeneous materials, through physically motivated and mathematically prescribed concepts such as inertial stress and heat flux, body and surface couples, and local angular velocity and time-evolving moment-of-inertia tensors. Whereas single-scale polar theories may postulate the existence and evolution of such quantities, the proposed approach relies on the underlying continuum-mechanical microscale and the proposed homogenization theory to constitutively specify them.

Acknowledgements. B.E. Abali's work was supported by a grant from the Max Kade Foundation to the University of California, Berkeley. K.K. Mandadapu is supported by the University of California, Berkeley.

\section{References}

[1] J.H. Irving and J.G. Kirkwood. The statistical mechanical theory of transport processes. IV. The equations of hydrodynamics. The Journal of Chemical Physics, 18:817-829, 1950.

[2] K.K. Mandadapu, A. Sengupta, and P. Papadopoulos. A homogenization method for thermomechanical continua using extensive physical quantities. Proceedings of Royal Society London 
A., 468:1696-1715, 2012.

[3] B. Mercer, K.K. Mandadapu, and P. Papadopoulos. Novel formulations of microscopic boundary-value problems in continuous multiscale finite element methods. Computer Methods in Applied Mechanics and Engineering, 286:268-292, 2015.

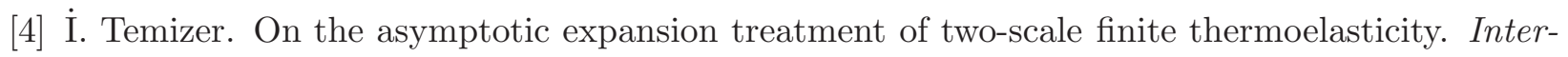
national Journal of Engineering Science, 53:74-84, 2012.

[5] M. Shen and W. Cao. Acoustic bandgap formation in a periodic structure with multilayer unit cells. Journal of Physics D: Applied Physics, 33:1150-1154, 2000.

[6] S. Nemat-Nasser and A. Srivastava. Overall dynamic constitutive relations of layered elastic composites. Journal of the Mechanics and Physics of Solids, 59:1953-1965, 2011.

[7] B. Mercer, K.K. Mandadapu, and P. Papadopoulos. Homogenization of high-frequency wave propagation in linearly elastic layered media using a continuum Irving-Kirkwood theory. International Journal of Solids and Structures, 96:162-172, 2016.

[8] G. Russakoff. A derivation of the macroscopic Maxwell equations. American Journal of Physics, 38:1188-1195, 1970.

[9] D. Mandal, K. Klymko, and K.K. Mandadapu. Generalized hydrodynamics of active polar suspensions. arXiv:1706.02284, 2017.

[10] K. Klymko, D. Mandal, and K.K. Mandadapu. Statistical mechanics of transport processes for active fluids: Equations of hydrodynamics. Journal of Chemical Physics, 147:194109, 2017.

[11] J.S. Dahler and L.E. Scriven. Angular momentum of continua. Nature, 192:36-37, 1961.

[12] A.C. Eringen and E.S. Suhubi. Nonlinear theory of simple micro-elastic solids. International Journal of Engineering Science, 2:189-203, 1964.

[13] A.C. Eringen. Mechanics of micromorphic continua. In E. Kröner, editor, Mechanics of Generalized Continua, pages 18-35. Springer-Verlag, Berlin, 1968.

[14] A.C. Eringen. Simple microfluids. International Journal of Engineering Science, 2(2):205-217, 1964.

[15] F.C. Frank. I. Liquid crystals. on the theory of liquid crystals. Discussions of the Faraday Society, 25:19-28, 1958. 
[16] R.D. Mindlin. Micro-structure in linear elasticity. Archive for Rational Mechanics and Analysis, 16(1):51-78, 1964.

[17] F.M. Leslie. Some constitutive equations for liquid crystals. Archive for Rational Mechanics and Analysis, 28(4):265-283, 1968.

[18] J.L. Lumley. Toward a turbulence constitutive relation. Journal of Fluid Mechanics, 41:413-434, 1970.

[19] J.L. Lumley. Turbulence modeling. ASME Journal of Applied Mechanics, 50:1097-1103, 1983.

[20] A. Sadiki and K. Hutter. On the frame dependence and form invariance of the transport equations for the Reynolds stress tensor and the turbulent heat flux vector: Its consequences on closure models in turbulence modelling. Continuum Mechanics and Thermodynamics, 8:341349, 1996.

[21] I. Müller. On the frame dependence of stress and heat flux. Archive for Rational Mechanics and Analysis, 45:241-250, 1972.

[22] C. Truesdell. Correction of two errors in the kinetic theory of gases which have been used to cast unfounded doubt upon the principle of material frame-indifference. Meccanica, 11(4):196-199, 1976.

[23] W.G. Hoover, B. Moran, R.M. More, and A.J.C. Ladd. Heat conduction in a rotating disk via nonequilibrium molecular dynamics. Physical Review A, 24:2109-2115, 1981.

[24] A. Bertram and R. Clüge. Solid Mechanics: Theory, Modeling, and Problems. Springer-Verlag, Cham, 2015.

[25] A.E. Green and P.M. Naghdi. A unified procedure for construction of theories of deformable media. i. classical continuum physics. Proceedings of the Royal Society of London A: Mathematical, Physical and Engineering Sciences, 448:335-356, 1995.

[26] B. Svendsen and A. Bertram. On frame-indifference and form-invariance in constitutive theory. Acta Mechanica, 132:195-207, 1999.

[27] S.-W. Chiu, M. Clark, S. Subramaniam, and E. Jakobsson. Collective motion artifacts arising in long-duration molecular dynamics simulations. Journal of Computational Chemistry, 21:121$131,2000$. 
[28] P. Chadwick. Continuum Mechanics: Concise Theory and Problems. Wiley, New York, 1976. 


\section{Appendix A Derivation of the Macroscopic Energy Balance Equa- tion}

To derive the macroscopic energy balance equation (35), start by taking the material time derivative of the extensivity equation (23) and then invoke (1) and (3) to find that

$$
\rho^{M} \dot{e}^{M}=\rho^{M} e^{M} \frac{\partial}{\partial \mathbf{y}} \cdot \mathbf{v}^{M}+\int_{\mathcal{R}} \rho^{m} \dot{e}^{m} g \mathrm{~d} v^{m}+\int_{\mathcal{R}} \rho^{m} e^{m} \dot{g} \mathrm{~d} v^{m} .
$$

The second term on the right-hand side of (A.1) may be expanded with the aid of the microscopic energy balance (21) and the definition of macroscopic body force (11) as

$$
\begin{aligned}
\int_{\mathcal{R}} \rho^{m} \dot{e}^{m} g \mathrm{~d} v^{m}=\rho^{M} \mathbf{b}^{M} \cdot \mathbf{v}^{M}+\int_{\mathcal{R}} \rho^{m} \mathbf{b}^{m} \cdot\left(\mathbf{v}^{m}-\mathbf{v}^{M}\right) g \mathrm{~d} v^{m}+\int_{\mathcal{R}} \rho^{m} r^{m} g \mathrm{~d} v^{m}+ \\
\int_{\mathcal{R}} \frac{\partial}{\partial \mathbf{x}} \cdot\left(\mathbf{T}^{m} \mathbf{v}^{m}\right) g \mathrm{~d} v^{m}-\int_{\mathcal{R}} \frac{\partial}{\partial \mathbf{x}} \cdot \mathbf{q}^{m} g \mathrm{~d} v^{m} .
\end{aligned}
$$

However, the divergence theorem, in conjunction with (7) and (9), implies that

$$
\int_{\mathcal{R}} \frac{\partial}{\partial \mathbf{x}} \cdot\left(\mathbf{T}^{m} \mathbf{v}^{m}\right) g \mathrm{~d} v^{m}=\frac{\partial}{\partial \mathbf{y}} \cdot \int_{\mathcal{R}} \mathbf{T}^{m}\left(\mathbf{v}^{m}-\mathbf{v}^{M}\right) g \mathrm{~d} v^{m}+\frac{\partial}{\partial \mathbf{y}} \cdot\left[\int_{\mathcal{R}} \mathbf{T}^{m} g \mathrm{~d} v^{m} \mathbf{v}^{M}\right]
$$

and

$$
\int_{\mathcal{R}} \frac{\partial}{\partial \mathbf{x}} \cdot \mathbf{q}^{m} g \mathrm{~d} v^{m}=\frac{\partial}{\partial \mathbf{y}} \cdot \int_{\mathcal{R}} \mathbf{q}^{m} g \mathrm{~d} v^{m} .
$$

Likewise, upon using (9) and (23), the third term on the right-hand side of (A.1) becomes

$$
\int_{\mathcal{R}} \rho^{m} e^{m} \dot{g} \mathrm{~d} v^{m}=-\frac{\partial}{\partial \mathbf{y}} \cdot \int_{\mathcal{R}} \rho^{m} e^{m}\left(\mathbf{v}^{m}-\mathbf{v}^{M}\right) g \mathrm{~d} v^{m}-\rho^{M} e^{M} \frac{\partial}{\partial \mathbf{y}} \cdot \mathbf{v}^{M} .
$$

Inserting (A.2) and (A.5) into (A.1), and taking into account (A.3), (A.4), and the definition of the macroscopic Cauchy stress in (10) leads to

$$
\begin{gathered}
\rho^{M} \dot{e}^{M}=\rho^{M} \mathbf{b}^{M} \cdot \mathbf{v}^{M}+\int_{\mathcal{R}}\left[\rho^{m} r^{m}+\rho^{m} \mathbf{b}^{m} \cdot\left(\mathbf{v}^{m}-\mathbf{v}^{M}\right)\right] g \mathrm{~d} v^{m}+\frac{\partial}{\partial \mathbf{y}} \cdot\left(\mathbf{T}^{M} \mathbf{v}^{M}\right) \\
-\frac{\partial}{\partial \mathbf{y}} \cdot \int_{\mathcal{R}}\left[\mathbf{q}^{m}-\mathbf{T}^{m}\left(\mathbf{v}^{m}-\mathbf{v}^{M}\right)+\rho^{m} e^{m}\left(\mathbf{v}^{m}-\mathbf{v}^{M}\right)\right] g \mathrm{~d} v^{m}+\frac{\partial}{\partial \mathbf{y}} \cdot\left[\int_{\mathcal{R}} \rho^{m}\left(\mathbf{v}^{m}-\mathbf{v}^{M}\right) \otimes\left(\mathbf{v}^{m}-\mathbf{v}^{M}\right) g \mathrm{~d} v^{m} \mathbf{v}^{M}\right] .
\end{gathered}
$$

To extract the polar effects from the preceding statement of energy balance, recall the definition of the convected microscopic velocity $\hat{\mathbf{v}}^{m}$ in $(25)$ and note that

$$
\int_{\mathcal{R}} \rho^{m} \mathbf{b}^{m} \cdot\left(\mathbf{v}^{m}-\mathbf{v}^{M}\right) g \mathrm{~d} v^{m}=\int_{\mathcal{R}} \rho^{m} \mathbf{b}^{m} \cdot\left(\mathbf{v}^{m}-\hat{\mathbf{v}}^{m}\right) g \mathrm{~d} v^{m}+\rho^{M} \mathbf{g}^{M} \cdot \mathbf{w}^{M},
$$

where use is made of (19). Likewise, it can be shown with the aid of (20) that 


$$
\begin{aligned}
\int_{\mathcal{R}} \mathbf{T}^{m}\left(\mathbf{v}^{m}-\mathbf{v}^{M}\right) g \mathrm{~d} v^{m}= & \int_{\mathcal{R}} \mathbf{T}^{m}\left(\mathbf{v}^{m}-\hat{\mathbf{v}}^{m}\right) g \mathrm{~d} v^{m}+\left(\mathbf{M}^{M}\right)^{T} \mathbf{w}^{M} \\
& +\left[\int_{\mathcal{R}}(\mathbf{x}-\mathbf{y}) \times\left[\rho^{m} \mathbf{v}^{m} \otimes\left(\mathbf{v}^{m}-\mathbf{v}^{M}\right)\right] g \mathrm{~d} v^{m}\right]^{T} \mathbf{w}^{M},
\end{aligned}
$$

Lastly, upon taking into account the definition of $\hat{e}^{m}$ in (36), hence its implied relation to the total internal energy $e^{m}$, the internal energy term in (A.6) may be expanded into

$$
\begin{aligned}
\int_{\mathcal{R}} \rho^{m} e^{m}\left(\mathbf{v}^{m}-\mathbf{v}^{M}\right) g \mathrm{~d} v^{m}=\int_{\mathcal{R}} \rho^{m} \hat{e}^{m}\left(\mathbf{v}^{m}-\mathbf{v}^{M}\right) g \mathrm{~d} v^{m} \\
\quad+\int_{\mathcal{R}} \frac{1}{2} \rho^{m}\left[\mathbf{v}^{m} \cdot \mathbf{v}^{m}-\left(\mathbf{v}^{m}-\hat{\mathbf{v}}^{m}\right) \cdot\left(\mathbf{v}^{m}-\hat{\mathbf{v}}^{m}\right)-\hat{\mathbf{v}}^{m} \cdot \hat{\mathbf{v}}^{m}\right]\left(\mathbf{v}^{m}-\mathbf{v}^{M}\right) g \mathrm{~d} v^{m} .
\end{aligned}
$$

The macroscopic energy balance equation (35) is obtained by substituting (A.7-A.9) into (A.6) and using (25) to eliminate all residual terms.

\section{Appendix B Divergence-free Terms in the Macroscopic Cauchy Stress}

Preliminary to establishing the divergence-free property of the additional inertial terms in (70), two useful identities are deduced. For the first identity, start by taking the material time derivative of the invariance relation (8), which yields

$$
\frac{\partial g}{\partial \mathbf{y}} \cdot \mathbf{v}^{M}+\frac{\partial g}{\partial \mathbf{x}} \cdot \mathbf{v}^{m}=\frac{\partial g}{\partial \mathbf{y}^{+}} \cdot \mathbf{v}^{M^{+}}+\frac{\partial g}{\partial \mathbf{x}^{+}} \cdot \mathbf{v}^{m+} .
$$

Next, upon invoking (40), (41), (43) and (44), equation (B.1) may be rewritten as

$$
\frac{\partial g}{\partial \mathbf{y}} \cdot \mathbf{v}^{M}+\frac{\partial g}{\partial \mathbf{x}} \cdot \mathbf{v}^{m}=\frac{\partial g}{\partial \mathbf{y}} \cdot\left(\mathbf{v}^{M}+\boldsymbol{\Omega} \mathbf{y}\right)+\frac{\partial g}{\partial \mathbf{x}} \cdot\left(\mathbf{v}^{m}+\boldsymbol{\Omega} \mathbf{x}\right)+\mathbf{Q}\left(\frac{\partial g}{\partial \mathbf{y}}+\frac{\partial g}{\partial \mathbf{x}}\right) \cdot \dot{\mathbf{c}}
$$

and further reduced, upon observing (9), to

$$
\boldsymbol{\Omega} \cdot\left(\mathbf{x} \otimes \frac{\partial g}{\partial \mathbf{x}}+\mathbf{y} \otimes \frac{\partial g}{\partial \mathbf{y}}\right)=0
$$

Given the arbitrariness of $\boldsymbol{\Omega}$, the preceding equation implies that the quantity in parentheses is necessarily symmetric. Furthermore, upon using again (9), equation (B.3) readily implies the first identity, in the form

$$
\boldsymbol{\Omega}(\mathbf{x}-\mathbf{y}) \cdot \frac{\partial g}{\partial \mathbf{y}}=0
$$

The second identity is obtained by taking the material time derivative of the center-of-mass relation (51). To this end, appealing to the Reynolds transport theorem and using the microscopic 
balance of mass (1), it follows that

$$
\frac{\mathrm{d}}{\mathrm{d} t}\left(\rho^{M} \mathbf{y}\right)=\int_{\mathcal{R}}\left[\rho^{m} \mathbf{v}^{m} g+\rho^{m} \mathbf{x}\left(\frac{\partial g}{\partial \mathbf{x}} \cdot \mathbf{v}^{m}\right)+\rho^{m} \mathbf{x}\left(\frac{\partial g}{\partial \mathbf{y}} \cdot \mathbf{v}^{M}\right)\right] \mathrm{d} v^{m}
$$

Using first (9) and then invoking (6) and (51), the preceding equation becomes

$$
\begin{aligned}
\frac{\mathrm{d}}{\mathrm{d} t}\left(\rho^{M} \mathbf{y}\right) & =\int_{\mathcal{R}} \rho^{m} \mathbf{v}^{m} g \mathrm{~d} v^{m}-\frac{\partial}{\partial \mathbf{y}} \cdot \int_{\mathcal{R}} \rho^{m} \mathbf{x} \otimes\left(\mathbf{v}^{m}-\mathbf{v}^{M}\right) g \mathrm{~d} v^{m}-\int_{\mathcal{R}} \rho^{m} \mathbf{x} \frac{\partial g}{\partial \mathbf{y}} \cdot \mathbf{v}^{M} \mathrm{~d} v^{m} \\
& =\rho^{M} \mathbf{v}^{M}-\frac{\partial}{\partial \mathbf{y}} \cdot \int_{\mathcal{R}} \rho^{m}(\mathbf{x}-\mathbf{y}) \otimes\left(\mathbf{v}^{m}-\mathbf{v}^{M}\right) g \mathrm{~d} v^{m}-\rho^{M} \mathbf{y} \frac{\partial}{\partial \mathbf{y}} \cdot \mathbf{v}^{M}
\end{aligned}
$$

Expanding now the left-hand side of (B.5), and using the macroscopic mass balance equation (3), it is concluded from (B.6) that

$$
\frac{\partial}{\partial \mathbf{y}} \cdot \int_{\mathcal{R}} \rho^{m}(\mathbf{x}-\mathbf{y}) \otimes\left(\mathbf{v}^{m}-\mathbf{v}^{M}\right) g \mathrm{~d} v^{m}=\mathbf{0}
$$

which is the second identity of interest here.

It is now possible to show that the last three terms on the right-hand side of (70) are individually divergence-free. Indeed, consider the first term, which takes the form

$$
\begin{aligned}
\frac{\partial}{\partial \mathbf{y}^{+}} \cdot\left(\mathbf{Q} \boldsymbol{\Omega} \mathbf{A} \mathbf{Q}^{T}\right) & =\mathbf{Q} \frac{\partial}{\partial \mathbf{y}} \cdot(\boldsymbol{\Omega A}) \\
& =\mathbf{Q} \boldsymbol{\Omega} \frac{\partial}{\partial \mathbf{y}} \cdot \int_{\mathcal{R}} \rho^{m}(\mathbf{x}-\mathbf{y}) \otimes\left(\mathbf{v}^{m}-\mathbf{v}^{M}\right) g \mathrm{~d} v^{m+}
\end{aligned}
$$

and vanishes identically due to (B.7). The next term is

$$
\begin{aligned}
\frac{\partial}{\partial \mathbf{y}} \cdot\left(\mathbf{Q}(\boldsymbol{\Omega} \mathbf{A})^{T} \mathbf{Q}\right)= & \mathbf{Q} \frac{\partial}{\partial \mathbf{y}} \cdot(\boldsymbol{\Omega A})^{T} \\
= & \mathbf{Q} \frac{\partial}{\partial \mathbf{y}} \cdot \int_{\mathcal{R}} \rho^{m}\left(\mathbf{v}^{m}-\mathbf{v}^{M}\right) \otimes(\mathbf{x}-\mathbf{y}) g \mathrm{~d} v^{m} \boldsymbol{\Omega}^{T} \\
= & \mathbf{Q} \int_{\mathcal{R}} \rho^{m}\left(-\frac{\partial \mathbf{v}^{M}}{\partial \mathbf{y}} \boldsymbol{\Omega}\right)(\mathbf{x}-\mathbf{y}) g \mathrm{~d} v^{m} \\
& \quad+\mathbf{Q} \int_{\mathcal{R}} \rho^{m}\left(\mathbf{v}^{m}-\mathbf{v}^{M}\right)(-\mathbf{i} \cdot \boldsymbol{\Omega}) g \mathrm{~d} v^{m} \\
& \quad+\mathbf{Q} \int_{\mathcal{R}} \rho^{m}\left(\mathbf{v}^{m}-\mathbf{v}^{M}\right)\left[\mathbf{\Omega}(\mathbf{x}-\mathbf{y}) \cdot \frac{\partial g}{\partial \mathbf{y}}\right] \mathrm{d} v^{m}
\end{aligned}
$$

The three terms on the right-hand side of (B.9) themselves vanish individually due to (51), the skew-symmetry of $\boldsymbol{\Omega}$, and the identity (B.4), respectively. Lastly, given the definition of B in (72), 
one may write

$$
\begin{aligned}
\frac{\partial}{\partial \mathbf{y}} \cdot\left(\mathbf{Q} \boldsymbol{\Omega} \mathbf{B} \boldsymbol{\Omega}^{T} \mathbf{Q}^{T}\right) & =\mathbf{Q} \frac{\partial}{\partial \mathbf{y}} \cdot\left(\boldsymbol{\Omega} \mathbf{B} \boldsymbol{\Omega}^{T}\right) \\
= & \mathbf{Q} \frac{\partial}{\partial \mathbf{y}} \cdot \int_{\mathcal{R}} \rho^{m} \boldsymbol{\Omega}(\mathbf{x}-\mathbf{y}) \otimes \boldsymbol{\Omega}(\mathbf{x}-\mathbf{y}) g \mathrm{~d} v^{m} \\
= & -\mathbf{Q} \int_{\mathcal{R}} \rho^{m} \boldsymbol{\Omega}^{2}(\mathbf{x}-\mathbf{y}) g \mathrm{~d} v^{m} \\
\quad-\mathbf{Q} \int_{\mathcal{R}} \rho^{m} \boldsymbol{\Omega}(\mathbf{x}-\mathbf{y})(-\mathbf{i} \cdot \boldsymbol{\Omega}) g \mathrm{~d} v^{m} & \quad \mathbf{Q} \int_{\mathcal{R}} \rho^{m} \boldsymbol{\Omega}(\mathbf{x}-\mathbf{y})\left[\boldsymbol{\Omega}(\mathbf{x}-\mathbf{y}) \cdot \frac{\partial g}{\partial \mathbf{y}}\right] \mathrm{d} v^{m} .
\end{aligned}
$$

Again, each of the three terms on the right-hand side of (B.10) vanishes owing to (51), the skewsymmetry of $\boldsymbol{\Omega}$, and the identity (B.7), respectively. Therefore, the last three terms on the right-hand side of (70) are individually divergence-free with respect to the macroscopic coordinates in the rigidly transformed frame. 


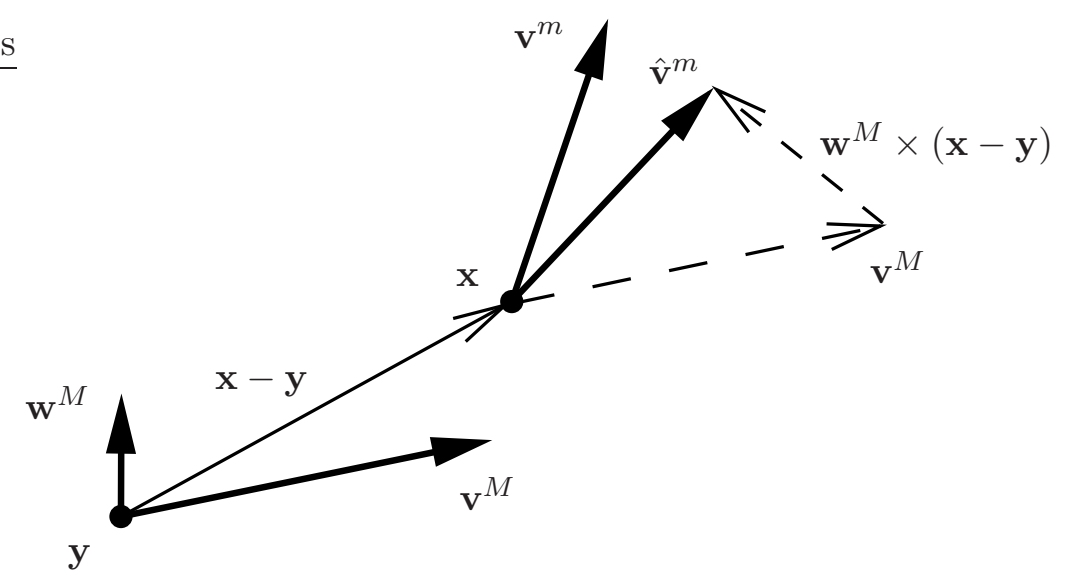

Figure 1: Schematic depiction of the velocities $\mathbf{v}^{m}, \hat{\mathbf{v}}^{m}$ and $\mathbf{v}^{M}$ at points with position vectors $\mathbf{x}$ and $\mathbf{y}$. 